\title{
MULLIN'S SEQUENCE OF PRIMES IS NOT MONOTONIC
}

\author{
THORKIL NAUR
}

\begin{abstract}
The sequence of primes defined by $p_{1}=2$ and $p_{n+1}=$ (largest prime factor of $\left.p_{1} \cdot p_{2} \cdots p_{n}+1\right)$ is not monotone increasing. We present the first eleven primes of the sequence and observe that $p_{10}<p_{9}$.
\end{abstract}

Following Euclid's scheme for proving the infinitude of the primes, Mullin [6] defines the sequence of primes

$$
p_{1}=2, \quad p_{n+1}=\text { largest prime factor of } p_{1} \cdot p_{2} \cdots p_{n}+1
$$

and asks (among other questions concerning this and a related sequence) whether it is monotone increasing. We have computed the first eleven terms of this sequence, which are given below (for completeness, we give all the known primes in the sequence):

$$
\begin{array}{rl}
n & p_{n} \\
1 & 2 \\
2 & 3 \\
3 & 7 \\
4 & 43 \\
5 & 139 \\
6 & 50207 \\
7 & 340999 \\
8 & 2365347734339 \\
9 & 4680225641471129 \\
10 & 1368845206580129 \\
11 & 889340324577880670089824574922371
\end{array}
$$

We observe that $p_{10}<p_{9}$ and the sequence is thus not monotone increasing.

Each $p_{n+1}$ was found by completely factoring $p_{1} \cdot p_{2} \cdots p_{n}+1$. The factorizations corresponding to the last four primes are

$$
\begin{array}{rl}
n & \text { Complete factorization of } p_{1} \cdot p_{2} \cdots p_{n}+1 \\
7 & 23 \cdot 79 \cdot p_{8} \\
8 & 17 \cdot 127770091783 \cdot p_{9} \\
9 & 89 \cdot 839491 \cdot 556266121 \cdot 836312735653 \cdot p_{10} \\
10 & 1307 \cdot 56030239485370382805887 \cdot p_{11}
\end{array}
$$

These factorizations were found using direct search and the methods described in Morrison and Brillhart [5] and Pollard [8, 9]. The larger prime factors were proved

Received by the editors January 21, 1983.

1980 Mathematics Subject Classification. Primary 10A40; Secondary 10A25.

(C)1984 American Mathematical Society 
prime by using the methods of Brillhart, Lehmer and Selfridge [1]. For details, see pp. 45-52 of Naur [7].

The first nine terms of the sequence, as published earlier in Guy and Nowakowski [3], agree with those given above. Guy and Nowakowski [3] also discuss several related sequences. The incorrect computation by Korfhage [4] is cited in Cox and van der Poorten [2] and Sloane [10] (sequence number 330).

Cox and van der Poorten [2] prove that if the $j$ th prime $q_{j}$ occurs as the $(k+1)$ st term of the sequence, then a certain set of congruences (modulo 2), which depends on $j$ and $k$, must be solvable. The congruences for $j=16, k=6$ are stated to be unsolvable, and this is taken as proving that none of the primes less than $q_{16}=53$ occur in the sequence, except for $2,3,7$, and 43 , which occur among the first $k=6$ terms. However, to prove the nonoccurrence of a prime using this argument, it is clearly necessary to show that the congruences are unsolvable for all $k$, not just one, so it is still not known whether all primes occur in the sequence. The unproved result concerning the nonoccurrence of primes less than 53 is also cited in Guy and Nowakowski [3].

The author would like to thank Professor B. H. Mayoh for bringing his attention to this problem and the referee for pointing out the paper by Guy and Nowakowski $[3]$.

\section{REFERENCES}

1. J. Brillhart, D. H. Lehmer and J. L. Selfridge, New primality criteria and factorizations of $2^{m} \pm 1$, Math. Comp. 29 (1975), 620-647.

2. C. D. Cox and A. J. van der Poorten, On a sequence of prime numbers, J. Austral. Math. Soc. 8 (1968), 571-574.

3. R. Guy and R. Nowakowski, Discovering primes with Euclid, Delta (Waukesha) 5 (1975), 49-63.

4. R. R. Korfhage, On a sequence of prime numbers, Bull. Amer. Math. Soc. 70 (1964), 341-342; Errata, 747.

5. M. A. Morrison and J. Brillhart, $A$ method of factoring and the factorization of $F_{7}$, Math. Comp. 29 (1975), 183-205.

6. A. A. Mullin, Recursive function theory, Bull. Amer. Math. Soc. 69 (1963), 737.

7. T. Naur, Integer factorization, DAIMI PB-144, Dept. Comput. Sci., Univ. of Aarhus, Denmark, 1982.

8. J. M. Pollard, Theorems on factorization and primality testing, Proc. Cambridge Philos. Soc. 76 (1974), 521-528.

9. __ A Monte Carlo method for factorization, BIT 15 (1975), 331-334.

10. N. J. A. Sloane, $A$ handbook of integer sequences, Academic Press, New York, 1973.

Department of Mathematics, Odense University, Odense, Denmark 